\title{
Editorial for the First International Conference on Energy-Aware High Performance Computing
}

\author{
Thomas Ludwig
}

Published online: 30 July 2010

(c) Springer-Verlag 2010

Energy consumption in high performance computing increased dramatically in the last decade and becomes a major obstacle for new installations of compute centres world wide. In Germany for each megawatt sustained power consumption costs of about one million Euros per year are estimated. In addition, the corresponding amount of 8.7 million $\mathrm{kWh}$ per year is an ecological problem independent of whether we use green electricity or not. In 2010 petaflops computers have an average power consumption of several megawatts. Current technology would not allow to build exaflops machines - they would need a nuclear power plant for the electricity and the electricity bill would be in the billion Euro range. However, looking at the Earth Simulator's data this argument would have been valid for petaflops computers in the early 2000 s.

The last years are characterized by a new trend in IT research that deals with ecology aware life-cycles: Green IT. Major efforts are undertaken to improve the energy efficiency of electronic components. Recently this trend is adapted in high performance computing. In the past HPC was considered as the Formula 1 of computing and that justified the absence of any mechanisms for power efficiency. Today we recognize more vendor and customer awareness for this issue: e.g. processors used in HPC exhibit power saving states similar to mobile processors. However, it will require several years until all abstraction layers of high performance computers are completely prepared for green IT.

T. Ludwig ( $\varangle)$

Fachbereich Informatik, Wissenschaftliches Rechnen, Universität

Hamburg, Bundesstraße 45a, 20146 Hamburg, Germany

e-mail: t.ludwig@computer.org
The goal of this newly established conference series is to bring researchers, vendors, and HPC centre administrators together to discuss the status and future of energy awareness in high performance computing.

Fields of interest cover all abstraction layers, from the lowest level of hardware technology, over operating system, compiler and application issues to facility technology like air conditioning, sensor technology and heat reuse. A comprehensive effort at all these layers is necessary to yield the overall energy reduction required to enable exaflops computers to be operational by the end of this decade-as predicted by the TOP500 list.

For this first conference on energy-aware high performance computing we received 17 scientific submissions out of which 10 were selected for publication in this special issue. The submissions cover several of the above mentioned abstraction layers and thus represent research efforts world-wide in high performance computing: hardware architecture, application issues, heterogeneous programming, job scheduling, and modeling and evaluation of energy consumption. This variety guarantees a good coverage of crucial research questions. This will trigger inspiring discussion during the two days of the conference. We hope to contribute to a successful cooperation between vendors and users of HPC equipment and foster more research in this field of green HPC.

The organizers express their gratitude to all contributors to this journal. Their research efforts will render high performance computing economically and ecologically aware. We thank the program committee members as well as all reviewers for their efforts in selecting an attractive content for this journal and for the conference.

Hamburg, September 2010 
Program committee members:

- Cosimo Anglano, Universita del Piemonte Orientale, Alessandria, Italy

- Wu-chun Feng, Virginia Tech, Blacksburg, Virginia, USA

- Laurent Lefevre, INRIA, Ecole Normale Superieure de Lyon, Lyon, France

- Dzmitry Kliazovich, University of Luxembourg, Luxembourg
- Matthias Müller, Technische Universität Dresden, Dresden, Germany

- Jean-Marc Pierson, Universität Paul Sabatier, Toulouse, France

- Erich Strohmeier, Lawrence Berkeley National Laboratory, Berkeley, California, USA

- Jordi Torres, Barcelona Supercomputer Center, Barcelona, Spain 